Please do not remove this page

RMIT

UNIVERSITY

\title{
Promoting construction workers' health: a multi-level system perspective
}

Lingard, Helen; Turner, Michelle

https://researchrepository.rmit.edu.au/esploro/outputs/9921860340701341/filesAndLinks?institution=61RMIT_INST\&index=null

Lingard, H., \& Turner, M. (2017). Promoting construction workers' health: a multi-level system perspective. Construction Management and Economics, 35(5), 239-253.

https://doi.org/10.1080/01446193.2016.1274828

Document Version: Accepted Manuscript

Published Version: https://doi.org/10.1080/01446193.2016.1274828

Repository homepage: https://researchrepository.rmit.edu.au

(C) 2017 Informa

Downloaded On 2023/04/27 00:15:29 +1000

Please do not remove this page 
Promoting construction workers' health: a multi-level system perspective

\author{
Helen Lingard and Michelle Turner
}

Centre for Work Health and Safety Research, RMIT University, Melbourne, Australia

\begin{abstract}
Construction workers suffer poor mental and physical health. To address this, organizations have implemented behavioural health promotion programmes. However, targeting workers' lifestyle behaviour without addressing relevant environmental factors is unlikely to produce significant or sustained improvement. An ecological perspective offers a different way of understanding the determinants of health, reflecting the interplay of factors at multiple levels and the dynamic, reciprocal and non-linear relationships between them. Qualitative data collected during focus groups and in interviews with workers and managers were analysed to explore factors impacting the effectiveness of a health promotion programme implemented at two work sites in Queensland, Australia. A qualitative causal loop diagram identifying determinants of construction workers' health behaviour was developed. The findings suggest that the adoption of healthy behaviours is influenced by factors operating at and between individual, family, workplace and industry levels. These factors suggest key leverage points that can be addressed in the design of future health promotion interventions for the construction industry. The research provides qualitative evidence of the need to consider workers' health in a holistic way and develop multi-level strategies to produce improved health behaviour and outcomes in the construction industry.
\end{abstract}

\title{
Introduction
}

\section{Aim}

At the 2008 meeting of the XVIII World Congress on Safety and Health at Work delegates signed a declaration recalling that the right to a safe and healthy working environment be recognized as a fundamental human right and recognizing that improving safety and health at work has a positive impact on working conditions, productivity and economic and social development (International Labor Office 2016). Many construction workers suffer from permanent work incapacity and are forced to stop working due to health problems before they reach the pension age (Arndt et al. 2005, Brenner and Ahern 2000, Siebert et al. 2001). Poor health and work disability among construction workers have been linked to occupational exposure to hazardous working conditions (Snashall 2005, Stocks et al. 2011) and psychosocial risk factors (Abbe et al. 2011). Recognition of the magnitude of the problem has led to the implementation of workplace health promotion programmes targeting construction workers (see, for example, Ludewig and Borstad 2003, Sorensen et al. 2007, Groeneveld et al. 2010). However, workplace health

promotion interventions sometimes produce short-lived or limited benefits (see, for example, the systematic review of worksite healthy eating programmes by Maes et al. 2015). In this paper, we draw on an ecological health perspective (underpinned by systems thinking) to understand the 
factors impacting workers' health at multiple levels, and consider context and environmental issues, as well as individual behavioural issues. The study draws on qualitative data collected during interviews and focus groups with workers to identify these factors and understand how workers perceive their impact. We suggest that asking workers what factors shape their health behaviours and outcomes (and how) provides an important new perspective on the relationship between workers' experiences in different life settings (e.g. family; the workplace; and the construction industry) and their health behaviour and outcomes (Ettner and Grzywacz 2001). We analysed qualitative data collected during the introduction of a workplace health promotion programme at two worksites in Queensland, Australia in order to:

- explore factors that workers and managers identified as having an impact on the effectiveness of the health promotion programme,

- specify dynamic hypotheses relating to the interactions and relationships between these factors and workers' behaviour and health and

- suggest leverage points that can be addressed in the design of future health promotion programmes for the construction industry.

We first review the literature pertaining to construction workers' health, before briefly describing the workplace health promotion programme implemented at the two worksites. We provide an overview and justification of our choice to utilize an ecological perspective to inform the analysis of the interview and focus group data. We describe the process we used for developing a causal loop diagram based upon the qualitative data, before presenting and discussing the results of the analysis. Finally, we conclude the paper by describing the opportunities presented by systems thinking for understanding the determinants of construction workers' health. We make suggestions about how this approach could inform the design of future health promotion programmes that target multiple levels of influence.

\section{The health of construction workers}

Construction workers are a high risk group for poor health and work disability (Welch 2009, Boschman et al. 2014). Over half of the occupational attributable cancer deaths in Great Britain are the result of exposures within construction (Rushton et al. 2008). Construction workers' exposure to ultraviolet light and chemicals makes them particularly prone to skin cancer (Pritchard and Dixon 2008). Construction workers experience elevated rates of contact dermatitis, all types of skin neoplasma, non-malignant pleural mesothelioma, lung cancer, pneumoconiosis and musculoskeletal disorders (Rushton et al., 2008). Construction is physically demanding and, in the USA, $40 \%$ of construction workers over the age of 50 experience chronic back pain (Dong et al. 2012). Construction work is also psychologically demanding and workers experience high levels of stress and burnout (Lingard et al. 2010, Bowen et al. 2014). Long work hours, typical of construction projects (Hannertz et al. 2005), are linked to cardiovascular disease, diabetes, illness leading to disability retirement, subjectively reported physical ill health and fatigue (Van der Hulst 2003). The incidence of mental distress among construction workers is twice the level of the general male population (Borsting Jacobsen et al. 2013) and construction workers experience a higher incidence of emotional/psychiatric disorders than other manual/non-managerial workers (Petersen and Zwerling 1998). Burnout, once thought to be a 'white collar' phenomenon, is prevalent and often contributes to early retirement in manual, non-managerial construction workers (Oude Hengel et al. 2012). Construction workers are also at high risk of suicide (Meltzer et al. 2008). In Australia, construction workers are six times more likely to die by suicide than through a workplace accident; 
and construction apprentices are two-and-a-half times more likely to suicide than other young men their age (http:// www.matesinconstruction.org.au, 2016).

\section{Workplace health promotion programmes}

Consistent with a focus on behaviour, health promotion programmes implemented in the construction industry have addressed issues such as: exercise to address shoulder pain (Ludewig and Borstad 2003); weight reduction to reduce the risk of cardiovascular disease (Groeneveld et al. 2010); smoking cessation and the consumption of fruit and vegetables (Sorensen et al. 2007); and physical exercise to improve aerobic capacity (Gram et al. 2012). However, it is often the case that workplace health promotion interventions only address one component part of the problem, i.e. workers' behaviour. Chu et al. (1997) note that many health promotion interventions are too narrowly focused on behaviour, drawing attention away from the socio-economic, environmental and organizational causes of poor health. A health promotion programme targeting the behaviour of construction workers at two worksites in the Queensland construction industry was evaluated by the authors. At these sites, initiatives were introduced including:

- a smoking cessation campaign,

- health-based information sessions and healthy food tasting sessions,

- a change of food options sold in the site canteen,

- a waist line measurement activity,

- yoga/stretching sessions and

- discounted gym memberships.

During the implementation of the programme, the authors used diary-based methods to measure health behaviour. The results produced no evidence of significant or sustained behaviour change (Lingard and Turner 2015). The current research presents the analysis of interview and focus group data collected at participating worksites to explore reasons for the limited impact of the behavioural health promotion interventions.

\section{Ecological perspectives in health research}

Our analysis was informed by an ecological perspective (Stokols 1992). Health researchers increasingly call for an ecological perspective in research and the design of health promotion interventions (see, for example, Sallis et al. 2008). Ecological perspectives use concepts derived from natural systems to help understand human systems and environments. Advocates of using an ecological perspective to understand health are driven by frustration with the focus on individualism associated with psychological approaches to disease prevention and health promotion. In contrast, an ecological perspective places increased focus on people's contexts, seeks to identify environmental constraints on behaviour and, thus, progress beyond a linear, mechanistic way of understanding the causes of poor health (McLaren and Hawe 2005). The need to consider context is partly driven by the acknowledgement that social and health inequalities are inextricably linked. For example, poor childhood conditions, low levels of education and blue-collar employment are consistently linked to unhealthy behaviours and psychosocial dispositions with negative health impacts in adult males (Lynch et al. 1997). An ecological health perspective is often underpinned by theories of human development that position individuals in nested settings that interact with one another. Within these settings, the concepts of interdependence, reciprocity and mutual interaction 
between people and their environments are fundamental to understanding how health is created or impacted (McLaren and Hawe 2005). For example, Bronfenbrenner (2009) suggests that individuals' development is impacted by their position at the centre of a set of nested interacting systems. Thus, individuals' health is influenced by their interactions with people in their immediate environments (microsystem). However, they are also impacted by the mesosystem, which refers to linkages and overlaps between settings in which individuals participate, such as work, family and community. Linkages between settings which an individual may not directly participate in, but that can have an impact on their health, such as social services, the mass media and politics, are described as the exosystem. All of these levels are embedded within a broader macrosystem, which includes the entire network of nested systems and has particular ideological, cultural and social characteristics. Proponents of an ecological perspective suggest that some individual or environmental conditions have a disproportionately high level of influence on individuals' health and well-being (Grzywacz and Fuqua 2000). Thus, they argue that health promotion interventions should be designed to address the most effective leverage points within these interacting systems that make up an individual's social environment (Kok et al. 2008).

One challenge associated with the adoption of an ecological perspective on health is that, rather than providing a single unified theory to explain and predict health outcomes, it provides an overarching framework within which different theories can be used to understand health. Grzywacz and Fuqua (2000) suggest that medium-range discipline-specific theories can be used to develop specific explanations about interactions between people and their environments, and their relations to health. We have adopted this approach in the current research.

\section{Systems thinking in health promotion}

Systems thinking is central to understanding health using an ecological perspective (McLaren and Hawes 2005) and has been used to examine and understand multi-level causes of health outcomes (Kreuter et al. 2004, Homer and Hirsch 2006). Systems thinking is particularly useful in identifying the non-linear, dynamic, reciprocal and time-delayed relationships between health risk exposures and outcomes (Galea et al. 2010, Mabry et al. 2013). Systems thinking has been widely used to understand the complex systemic issues that affect health, with a view to developing intervention strategies that go beyond simple symptomatic prevention measures (Ritchie-Dunham and Méndez Galván 1999, Naaldenberg et al. 2009, Peters 2014). System dynamics models have been used to explain or predict complex social processes to provide a holistic understanding of the relationships between the determinants of health and understand the multi-level factors that can prevent illness or promote good health (Homer and Hirsch 2006). Topic areas have included underage drinking in high school children (LeBlue et al. 2012), HIV prevention (Weeks et al. 2013), asthma prevention (Gillen et al. 2014), cardiovascular disease prevention (Hirsch et al. 2010) and the prevention of Dengue fever (Ritchie-Dunham and Méndez Galván 1999). Developing system dynamics models involves the generation of dynamic hypotheses reflecting causal relationships and feedback loops. These relationships are then represented diagrammatically and can be subject to quantitative testing and analysis (Homer and Hirsch 2006). However, in other instances, a mixed methods or purely qualitative approach is adopted in the formulation and description of contextual factors and processes that interact to shape health in a particular setting (Weeks et al. 2013). 


\section{Qualitative system dynamics}

The use of qualitative approaches to analysing system dynamics is contentious. Wolstenholme (1999) describes an 'evolving balance' between quantitative and qualitative approaches to system dynamics modelling. Wolstenholme and Coyle (1983) argue that qualitatively derived causal loop diagrams - used to represent the feedback loops and dynamic behaviour of a system - can assist in issue-structuring and problem-solving without computer simulation. The use of qualitative approaches to guide problem structuring is particularly helpful when applied to messy problems, i.e messy problems, i.e. those situations in which 'there are large differences of opinion on the problem or even on the question of whether there is a problem' (Vennix 1999, p. 380). Vennix (1999) argues that qualitative modelling approaches add rigour to the analysis of complex issues, and help decision-makers to understand feedback loops and behaviour when designing strategies to deal with problems. Coyle (2000) strongly argues against the view that quantitative system dynamics models are always better than qualitative models. Coyle (2000) argues that quantitative simulation models can be characterized by so much uncertainty that drawing policy inferences from them can sometimes be dangerous. This is particularly the case when 'soft' variables are quantified for modelling purposes. When multiple soft variables are included in models, then Coyle suggests the problem can be compounded to the extent that inferences drawn from quantitative modelling may be no more useful than those drawn from qualitative approaches. Other writers disagree, arguing that quantitative modelling always adds value to policy analysis beyond that achieved by qualitative analysis (Homer and Olivia 2001). Coyle (2001) recommends further research to investigate whether quantification always produces better 'value for money' in system dynamics modelling and the discussion is far from resolved. Notwithstanding this, there is a well-established tradition of using purely qualitative models to structure issues, analyse causes and guide problem-solving, including in the field of health research (Gillen et al. 2014).

\section{Methods}

\section{Research framework}

The data were collected during a research project in which various health promotion strategies were implemented and evaluated in the construction industry in Queensland, Australia. During the research, data were collected at two separate worksites, under the control of the same contracting organization. The organization is one of Australia's largest providers of construction and property maintenance services. The research was undertaken as part of the 'Healthier. Happier. Workplaces' programmes, an initiative of the Queensland Government designed to support workplaces to implement programmes that improve the health and well-being of workers. The scheme specifically focused on five health risk factors (i.e. smoking, poor nutrition, excessive alcohol intake, physical inactivity and obesity). Thus, the research involved examining workers' health and lifestyle behaviours in relation to these five areas and we followed the implementation of health promotion interventions at the two worksites.

\section{Data collection}

In this paper, we draw on the qualitative (focus group and interview) data to develop a system dynamics model to represent variables impacting workers' health behaviour during the intervention 
period. Focus groups were undertaken at the two sites following the implementation of health promotion activities. These post-implementation focus groups explored workers' use of health promotion activities, as well as their perceptions relating to the effectiveness and impact of these activities. At the larger of the two sites (site 1), this workshop was supplemented with individual indepth interviews with a sample of workers to explore, in particular, perceptions of factors that can promote good health, as well as barriers to the adoption and impact of health promotion activities. Interviews could not be conducted at site 2 because during the research, the organization's contract for services at the site came to an end. The organization did not win a bid for continuation of its services at the site and workers were not available for interview after this point in time. Finally, a focus group was held with managers and occupational health and safety professionals in the organization's head office (in Sydney) to explore factors that, in their opinion, could improve the use and effectiveness of health promotion programmes in the Australian construction industry.

\section{Model building process}

A stepwise method for system description and the development of meaningful system diagrams was used to analyse the qualitative data (Wolstenholme and Coyle 1983). Wolstenholme and Coyle (1983) developed a set of rules for the development of systems models depicted as influence diagrams. First, key resources and their varying states relevant to the observed problem symptoms were identified. In the case of our research, the relevant symptom variables were the state of the workforce's health and the rates of healthy behaviours (that the health promotion intervention sought to change). Next, the forces that influence or change resources between different states (for example, good or poor health, low or high adoption of healthy behaviours) were identified by systematically working outwards from these symptom variables until an appropriate boundary was reached. Lastly, influence relationships and feedback loops responses indicated by the qualitative data were identified and superimposed on the diagram, depicted as solid black lines with arrows indicating the direction of influence. These lines represent dynamic hypotheses that can be tested in subsequent qualitative or quantitative research. Ecological theorists acknowledge that the boundaries of social ecosystems that impact on health are unlikely to be clearly defined (McLaren and Hawe 2005). However, Wolstenholme and Coyle (1983) suggest that developing a qualitative system dynamics model in a controlled way is useful in determining an appropriate boundary for a particular system. In our analysis, the boundary was defined by the experiences and conditions that workers reported as impacting their health behaviour or their health.

\section{Data analysis}

The qualitative focus group and interview data were subjected to inductive thematic analysis (Joffe and Yardley 2004) to: • identify the conditions and experiences that workers identified as supporting or impeding them from changing their health-related behaviour or experiencing a state of good health; • establish influence relationships and feedback loops through which these conditions and experiences influence health behaviour or health and $\bullet$ develop a qualitative model reflecting the system forces affecting the uptake and impact of the health promotion activities and state of workforce health. The data were independently analysed by two researchers who agreed on the emergent themes, thereby ensuring interrater reliability (Ballinger, Yardley and Payne 2004). 


\section{Results}

\section{Participating worksites}

Site 1 was a $\$ 1.8$-billion 738 bed public hospital which was constructed over four years. The site is based on the outer fringe of an urban area. The site had a canteen which sold healthy food and site sheds had basic facilities for meal preparation. At the time of the research, there were approximately 240 workers engaged at this site.

Site 2 was a power station at which a contractor was engaged to provide maintenance services. The site was located approximately 30 kilometres from the nearest township and the closest food vendors were located in the township. There were basic food preparation facilities at the site. At the time the research was undertaken, the site team consisted of 27 members.

\section{Health promotion activities}

Each worksite independently developed health promotion activities during the research. These were negotiated and decided upon by managers and workers at each site. Activities at site 1 included: (1) the introduction of a smoking cessation programme that was available to workers and their family members, (2) a healthy eating promotion programme that included food tasting, cooking instruction and the provision of healthy food options in the site canteen and (3) the introduction of on-site yoga and stretching sessions. At site 2, a competitive programme was introduced to encourage workers to become more physically active. In addition to this, fresh fruit was delivered to the site on a weekly basis.

\section{The sample}

Participation in the qualitative data collection is shown in Table 1. In total, 41 workers participated in focus groups (including 22 at site 1, 8 at site 2 and 11 in the organization's head office). Twelve workers participated in in-depth interviews at site 1.

Table 1. Participants in the qualitative data collection.

\begin{tabular}{|l|l|}
\hline Site & Participants \\
\hline Site 1 & $\begin{array}{l}\text { 22 workers participated in a focus group session to explore responses to the } \\
\text { interventions implemented at site 1 } \\
12 \text { workers participated in in-depth interviews to explore factors impacting on the } \\
\text { uptake and impact of the health promotion interventions }\end{array}$ \\
\hline Site 2 & $\begin{array}{l}\text { 8 workers participated in a focus group session to explore responses to the } \\
\text { interventions implemented at site 2 }\end{array}$ \\
\hline $\begin{array}{l}\text { Head } \\
\text { office }\end{array}$ & $\begin{array}{l}\text { office } 11 \text { managers and work health and safety professionals participated in a focus } \\
\text { group session to explore the findings relating to the uptake and impact of the } \\
\text { interventions at sites 1 and 2 }\end{array}$ \\
\hline
\end{tabular}




\section{Emergent themes and relevant factors}

The qualitative data were analysed to identify conditions or experiences that could be linked to health behaviour or health outcomes. This analysis was informed by an ecological framework in which factors of relevance were explored across four levels. These were: (1) workers' personal characteristics and motivations; (2) workers' experiences and interactions with family members; (3) workers' experiences and interactions at work; and (4) workers' experiences of working in the construction industry. Previous research suggests that individual characteristics interact with experiences in different life settings (e.g. family, workplace and society) to shape health outcomes (Grzywacz and Fuqua 2000). We sought to identify leverage points that could effect change at multiple levels. Variables identified as being important at each of these levels are shown in Table 2 . The data were further analysed to identify interactions and relationships between these variables of relevance (and health behaviour/outcomes) to provide insight into the systems forces that might explain the success or failure of health promotion activities at the two worksites.

Figure 1 is an illustrative system dynamics causal loop diagram produced as a result of this analysis. The rectangular boxes represent 'stocks' of construction workers who are using health promotion strategies implemented and who are healthy and well. The thick arrows represent the anticipated flow of health in the workforce, as the number of workers using health promotion strategies increases, so too does the number of healthy construction workers. The clouds displayed at the beginning of the inflow and the end of the outflow represent stock boundaries. The thin arrows represent causal links identified in the analysis. These represent dynamic hypotheses that can be tested in future qualitative or quantitative studies. Those links labelled ' $s$ ' are hypothesized to be related positively, i.e. the variables go up or down in the same direction. Those labelled 'o' are hypothesized to be related inversely, i.e. the variables change in the opposite direction to one another. Those labelled ' $R$ ' are hypothesized to be related to reinforcing feedback loops (these are discussed in more detail later). Consistent with ecological health theories, the data suggest that the rate of adoption of healthy behaviours by construction workers is influenced by multiple factors operating at and between the individual, family, workplace and industry levels. These dynamic relationships are discussed below.

\section{Individual-level influences}

At the individual level, some workers expressed a desire to be healthy and indicated that this influenced their behaviour. For example, one commented in relation to healthy eating behaviour:

I have been trying to be good at the canteen. The canteen has some good salads. I do the fruit once every two weeks, grab some fruit on the way. My eating habits have changed. My girlfriend and I have started eating healthy at home.

Another indicated he had cut back his alcohol consumption and also improved his diet:

I have been on a detox looking after myself. I am eating the best I have ever eaten. I have got eczema, I thought I would try eating right. I am sick of waking up hungover. I bring my own lunch. Save $\$ 80$ per week - beer money on the weekend. Cook my own dinner. Hangovers are the worst. Probably only drink 2-3 stubbies during the week and feel better for it. 
However, other workers indicated that they had no desire or intention to be healthier. One commented:

Yes. I enjoy it - having a smoke and a drink. I once had a job where they encouraged us to stop and have a smoke before jumping into a job, [it] makes you think about it. It does work. You have to die of something. I am going to enjoy myself while I am here.

Another quipped, 'I heard that red wine has more antioxidants so I have stopped drinking a couple of beers and now drink a couple of bottles of red wine'.

Even when suffering from serious health conditions, some workers indicated a low intention to improve their health. For example, one commented:

I have an enlarged liver and emphysema. I had a hole in my bowel last year. Everything else is selfinflicted. I am 50 in a couple of months. Our lifestyle catches up on us. No-one is going to stop me, it is my body'.

Table 2. Social ecological framework.

\begin{tabular}{|l|l|l|l|}
\hline Individual & Family & Workplace & Industry \\
\hline $\begin{array}{l}\text { time available for } \\
\text { healthy behaviours }\end{array}$ & $\begin{array}{l}\text { choice to spend time } \\
\text { with family }\end{array}$ & $\begin{array}{l}\text { long work hours } \\
\text { (combined with long } \\
\text { commute times) }\end{array}$ & $\begin{array}{l}\text { client-imposed } \\
\text { schedule demands }\end{array}$ \\
\hline $\begin{array}{l}\text { fatigue and physical } \\
\text { tiredness }\end{array}$ & $\begin{array}{l}\text { family support for } \\
\text { health }\end{array}$ & $\begin{array}{l}\text { concerns about job } \\
\text { security }\end{array}$ & $\begin{array}{l}\text { masculine work } \\
\text { culture }\end{array}$ \\
\hline $\begin{array}{l}\text { intention to be } \\
\text { healthier }\end{array}$ & $\begin{array}{l}\text { priority placed on } \\
\text { family relationships }\end{array}$ & $\begin{array}{l}\text { impact of physical } \\
\text { work }\end{array}$ & Project-based work \\
\hline
\end{tabular}




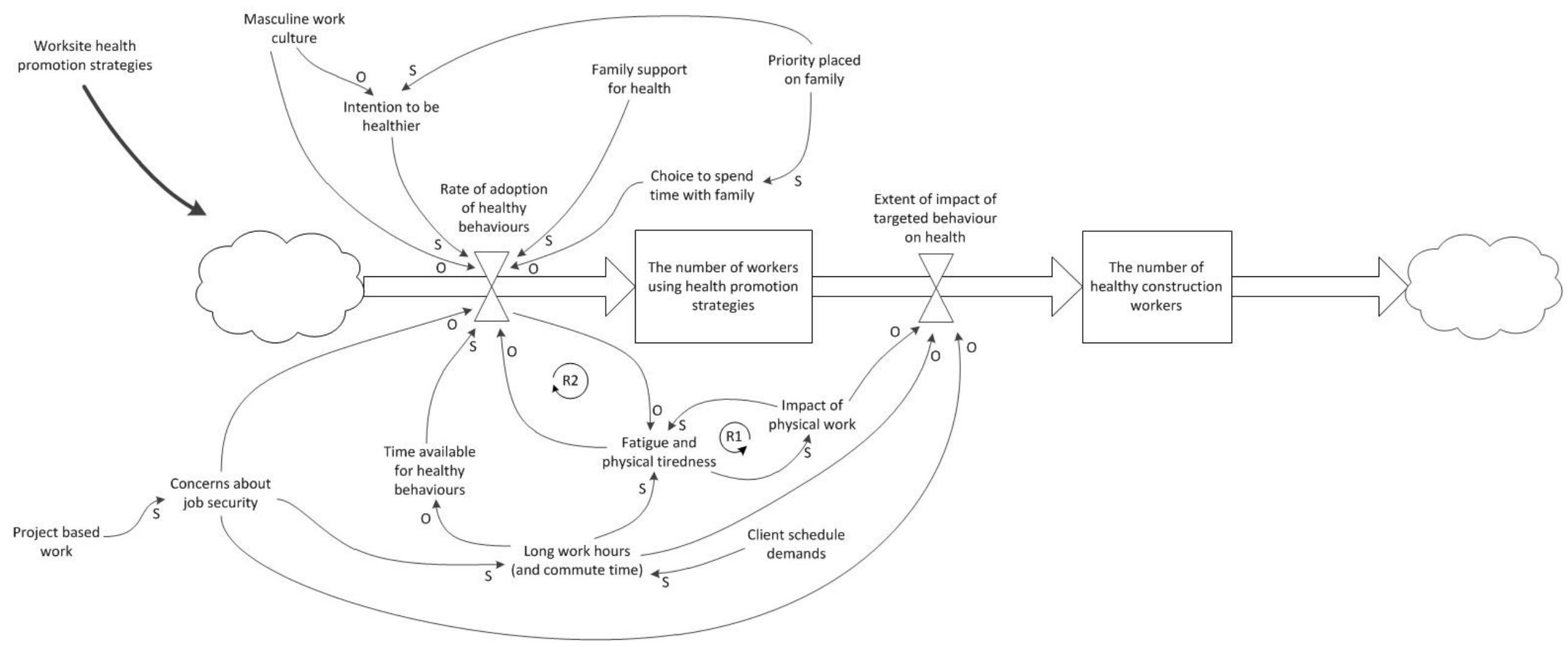

Figure 1: Illustrative causal loop diagram of the causal determinants of construction workers' health-related behaviour, health and wellbeing. 


\section{Family-level influences}

The data suggest that workers' intention to improve their health was often driven by family, in particular the experience of the death of a family member or a desire to be healthy so that they could enjoy their children as they grow up. For example, one worker explained the reason he wanted to be healthier:

My daughter - as soon as she was born. I do everything for her now. Motivation is for her now. I am a lot calmer since she came along. I used to have a short fuse. Now I have a much longer fuse. She gets up around 5 am and we hang out till I leave for work at 6 am.

Another reflected:

I have seen a lot of relatives die young, would like to see my kids grow up. I have had four uncles die of cancer and two aunties. I have a couple of health dramas so have to stay on top of it. With kids if we are eating rubbish, they will eat rubbish.

In some instances, family members had asked their construction worker relatives to improve their health. One older worker, whose wife had died and who had adult children, described how 'My son told me to cut back on the grog [alcohol]'.

Several workers also indicated that the priority they placed on family led them to choose to spend their limited time away from work with their family members. This choice meant that they actually had less time to engage in healthy activities, such as physical exercise and relaxation. For example, one commented, 'My time at home with my wife is limited so I choose to spend it with her. She can't walk as far as I can so we just stay home'. Another reflected that 'When you have children it is either you or them. For me to go home and say I want to train [at the gym], that's a big impact on the family'.

These comments indicate that when workers place a high priority on their family, this can have both a positive and a negative influence in relation to their engagement with health promotion activities, particularly if these activities require workers to commit their scarce non-work time to these activities.

Family support was also identified as an enabler of workers' adoption of healthy behaviours, particularly in relation to the consumption of healthy food. Workers frequently commented that their domestic partners played a key role in determining the food that they ate, preparing nutritious meals and providing healthy packed lunches for consumption during the work day. For example, one worker commented:

'My wife definitely is a great support. [I] would be a lot worse off if I didn't have her'.

Another stated: 'I eat pretty good. My wife cooks my meat and veggies [vegetables] every day and a good lunch. I have fruit in my lunch box every day ... might not eat it, but it is there. She looks after $m e^{\prime}$.

\section{Workplace-level influences}

Workers described how the long work hours they spent at work (including time spent commuting to and from work) impact their lifestyle. One described his day as follows: 
'II leave home at 4.45 am because I kick off in the mornings. By the time you come home, feed the dog, clean the house, wash your clothes, eat, that's it'.

The long hours of work also significantly reduce the time available to participate in healthy activities. For example, one worker described how:

I don't have time to go for a walk. When I lived in town (closer to work) I'd get up at 4:30 am and walk for an hour. Now if I did that l'd get up at 2:30 am (because of extra travel time). I just wouldn't have any time to sleep.

Another commented: Time is the biggest barrier. If you don't have the time, you don't have the time. If you want to do something extra in your day, you will be doing it before you go to work in the dark. By the time you get home, you are exhausted and just want to sit down, you don't want to do anything.

Time was a key factor influencing health behaviour among the workers who participated in the focus groups and interviews. One commented on the trade-offs that are sometimes made between sleep and exercise as follows:

It is a time thing, some guys go to the gym at 3.30 am in the morning. That is what you have to do in this industry, something has to be sacrificed. Sleep time gets traded. You end up brain dead. We used to have sacred Saturday - do an 8 hour day and have Mondays off and then come back to work on a Tuesday. That allowed you to get your jobs done - like going to the doctor or having lunch with your wife but that got traded and you don't do those things now. If you don't get everything done, it is a sense of under achieving.

This quotation also indicates the challenges these workers face in maintaining healthy family relationships and seeking help for medical problems should they arise. The time demands imposed on construction workers were perceived by the workers as being related to the unrealistic expectations of the principal contractor. For example, one worker commented:

'Time - the pressure from [the organization] is enormous'.

However, participants in the focus group with managers and occupational health and safety professionals conducted in the organization's head office noted the role played by clients in driving tight schedules, impacting workers' health. For example, one manager commented:

If our client wanted us to build a road in a particular way and they wanted us to invest in the health and wellbeing of our employees and they were able to compensate us through that process to enable us to do that, we would do it and we would make sure we do it because we have to do it. If large scale clients of that nature make the decision that they want health and wellbeing of the workforce to be a priority then the industry will follow suit ... and that comes into work hours, it comes into travel distances, it comes into all sorts of other things.

When asked how to address the long hours culture of the industry, the manager responded simply:

Five day working week in a contract, mandated. It's priced that way, it's built that way ... It would be brilliant. The five day working week ... if we actually had clients who were saying this is how we want you to work, would make our lives so much easier.

Job security concerns associated with project-based work were also prevalent in the comments of workers at both sites. For example, one commented: 
You don't have any job security. For me when the cranes come down, I don't have a job and work casually till the cranes go back up. Everyone worries. Insecurity affects everyone on site. The first crane goes by Christmas, those guys start to worry how long before they get work again.

These job security concerns were identified as being a driver of long work hours, as workers willingly worked long hours (including significant amounts of overtime) in order to maximize the money they earned from a job. One worker explained:

'We have to make the money whilst we are here and everyone wants the overtime. It comes down to the individual' and another said 'it's not going to change, money is a motivation, not going to change'. Workers' concerns about their job security were also directly linked to health behaviour, particularly at site 2 , at which workers were advised that their contract for maintenance services had not been renewed by the client. At this site, workers attributed job security concerns to increased alcohol consumption, one saying: 'I only had 2 drinks last week and none so far this week (but it's only Wednesday). But the week before I was having 3 drinks a night every night (due to the stress)'.

Another worker explained his low level of physical exercise saying: 'You need to have your head in the right space to exercise'.

Comments made by workers at site 2 suggest that their extreme concerns about job security also produced indications of mental distress. One worker described how:

When we found out the contract was up for renewal they told us you're never going to win. Then they got our hopes that we would win. Then we got told we didn't win it. It was like being in a tumble dryer!

Another commented on the impact of the announcement as follows:

'The announcement [that the contract would not be renewed] had a huge effect on everyone. It changed how we think and how we react to everything'.

Finally, the long hours and physical impact of work on workers' health were evident in some of the comments made by workers. One worker explained: 'I can't do the yoga stretching - I have too many injuries'. Another commented: 'I don't have time to be healthy - I made it to 50!' These comments reveal a resignation among the construction workers to the fact that they will experience poor health as they age. This acceptance of poor health as an inevitable aspect of working in the construction industry is likely to impede the positive impact of health promotion programmes.

\section{Industry-level influences}

Comments reflecting the importance of family support in adopting healthy eating habits highlight the gendered nature of the construction industry and the division of domestic labour in these workers' households. No female construction workers participated in our focus groups or interviews, reflecting the low representation of female workers in manual site-based construction roles at the two sites, and in the Australian construction industry generally. It is therefore impossible to determine whether the family support for healthy behaviour would be identified as a causal factor in the system of variables shaping female workers' health behaviour and outcomes.

The data suggest that workers' intention to be healthier was also influenced by the masculine work culture that prevails in the construction industry. In particular, the attitude that 'you only live once' was evident in comments made during the interviews and focus groups. One worker commented: 
Yes, have smoked for 40 years - one pack a day for 40 years. Went on a pipe for a while - 3 to 4 pipes a day, then start drinking and started smoking again. But I'm off the smokes at the moment because of the rib injury. I like smoking. You have to live while you can and enjoy it.

The same worker went on to describe his routine for his only day off: 'On a Sunday I sleep in to 6 am. I might have my first beer at 8 am then maybe another beer at midday'.

The masculine work culture shaped workers' use of some of the health promotion strategies implemented, particularly the yoga and stretching sessions provided at site 1 . Several workers at this site commented that: 'There are over 400 blokes here. Call it pride but there is no way in hell are you going to get me involved with yoga'.

Another expressed the view that the workers: Don't really pay any attention to it [the health promotion program]. I think what I do is right. [You] would probably cop too much shit if you went to yoga. The motivation is the boys you are working with, the job site and the management. If that is working you are happy to go to work.

The self-perpetuating nature of the industry culture was also evident in the following comment offered by one supervisor explaining the low take-up of the health promotion strategies:

The culture in the industry - the young guys follow the older guys for years and then make changes. It's the culture on the job site. A lot of guys go straight to the pub. My guys are different - we go fishing or to the beach if we finish a bit earlier.

It is noteworthy that this supervisor identified his personal leadership as a point of difference impacting his team members' choice to engage in healthy activities after work, as opposed to what the supervisor believes is the 'norm' behaviour of going to the pub. The adoption of healthy behaviours in response to the health promotion strategies was also influenced by workers' experiences of fatigue and physical tiredness. In particular, this impacted their engagement in physical activity outside work. For example, one worker described how:

If you work in the sun all day with concreting and scaffolding ... the last thing you want to do is go home and go to the gym because you have been out there all day. Half the time you don't get lunch. You don't want to be more physical.

However, this relationship seems to be part of a vicious cycle, indicated by reinforcing feedback loops R1 and R2. The data suggest that when they are tired or fatigued, workers engage in less physical exercise and poor eating habits. This, in turn, results in the deterioration of physical fitness which increases the impact of work on increasing subsequent levels of tiredness and fatigue. This cyclical effect is reflected in the following comment, which shows how unhealthy patterns of behaviour can become self-perpetuating:

You get into a cycle. There's not enough time. It's hard to step back and make a change in your lifestyle. You get into a pattern of eat, smoke, drink, sleep. Then you wake up and do it all again. Before you know it you have put on 20 kilos.

At the same time, workers who are fatigued and tired need to have sufficient time to recover between work shifts. One worker commented: 
When I do long hours of 9.5-10 hours then travel, (while) I'm tired from the physical work I'm also mentally tired. My body wants to get into wind down mode. I'm not ready for another peak from physical exercise.

However, if recovery is insufficient, the amount of effort needed to accomplish the same amount of work increases, resulting in increased levels of tiredness and fatigue.

\section{Discussion}

\section{Health inequality and construction workers}

Many well-intentioned information-based health promotion programmes cast construction workers as being 'victims of their ignorance'. The assumption is that, equipped with knowledge about the benefits of a healthy lifestyle, behaviour will change. However, our results indicate that this is not the case.

Our results confirm some underlying structural impediments to construction workers' adoption of healthy promotion strategies and health behaviour changes. The construction workers in our sample expressed a sense of disempowerment and resignation to the likelihood of poor health as they age. A similar finding was observed by Kolmet et al. (2006) who used a socio-ethnographic approach to understand determinants of health among Australian male blue-collar workers. Kolmet et al. (2006) report that men's health expectations were low due to anticipated 'wear and tear' caused by the physical demands of their work, stress inherent in balancing work and family demands and overall lifestyle.

Consistent with this finding, the construction workers in our sample experienced physical injuries and bodily pain as a result of their work but seemed to accept this as an inevitable part of working in construction. They also described how they struggled to meet work and family demands and adopted unhealthy lifestyle choices, despite knowing that these were detrimental to good health. The implication of these findings is that prevention strategies that address the underlying and structural causes of poor health in the construction context are needed. Ajslev et al. (2013) similarly describe how the fast pace and 'brutal rhythms' of construction work reproduce a working-class masculinity in which strain and bodily pain are seen as inevitable conditions of work. Our causal loop diagram reflects that client demands contribute to an intensification of work that produces time poverty, fatigue and physical impacts over time.

Our data also indicate some workers felt powerless to change their health yet, paradoxically, these workers also expressed individual responsibility for their unhealthy behaviour. Understanding the impact of the social organization of construction work on health is critical to addressing and changing health outcomes. In particular, our analysis identified client behaviour and the organization of project-based work as being a key leverage point for change.

Kolmet et al. (2006) report traditional gender role expectations are linked to unhealthy lifestyle choices and poor health. They found that Australian men in blue-collar job roles would like to participate more in family life. The workers in our sample described how they did not have 'time to be healthy' and commented on how valuable and important their limited family time was to them. Although the workers in our sample expressed the desire to live a more healthy and balanced life, long hours of work in the construction industry remain a significant impediment to this. Research 
shows significant health benefits flowing from family processes and social interactions (Bolin et al. 2003). The hours of work required in project-based construction work not only militate against gender equality but also have a negative impact on all workers' ability to participate fully in family life. Structures of work perpetuate traditional gendered breadwinner-homemaker arrangements. Thus, our findings identify working time arrangements and the affordance of a healthy work-family relationship as a key leverage point for improving construction workers' health.

\section{Individual effects on workers' health}

The research confirms that construction workers' individual motivations and health beliefs have an impact in shaping their health-related behaviours. We acknowledge that these are influenced by issues of socio-economic status and assumptions about work and life discussed above.

However, the workers in our sample differed in terms of their behaviour. While some of the workers in our sample were resigned to poor health (see above) and expressed a sense of powerlessness to make positive health-promoting lifestyle changes, others indicated they had consciously changed their behaviours to improve their health, for example by changing eating habits or engaging in a routine of regular physical exercise outside work.

The transtheoretical model of health behaviour posits a heuristic progression of stages of change. This describes a sequence of steps in successful behaviour change from: pre-contemplation (no recognition of the need for change); contemplation (thinking about changing); preparation (planning for change); action (changing habits); and maintenance (ongoing practice of healthy behaviour) (Glanz and Bishop 2010). Our results could reflect the fact that workers in our sample varied in terms of where they were situated in relation to these stages of change. It is possible that some workers we interviewed had reached the action stage, while others remained in the pre-contemplation stage. One limitation associated with our research was the lack of follow-up. Thus, we have no way of assessing the extent to which healthy behaviour was maintained by those who indicated that they had consciously made changes. Our causal loop diagram reflects the role played by individual's personal intentions to be healthier, which is sometimes driven by family considerations. Consequently, individuals' individual intentions with regard to their health and health behaviour should be considered a key leverage point for change. However, in the context of the masculine work culture and social context of work, further research is needed to identify key points of difference between workers transitioning between the stages of behaviour change, as well as potential structural barriers to these transitions.

\section{Environmental effects on workers' health}

Consistent with the social ecological perspective taken in this research, we also identified environmental factors that workers perceived had an impact on their health-related behaviour and/or health. These environmental factors were linked to experiences and conditions in the microsystems of the workplace and family and the mesosystem representing the overlap between these two life domains.

Workers in our sample described family as being a health-promoting support, as well as a source of demands that limited time available for healthy behaviour. The impact of family structure on health has been studied widely. Historically, being married was reported to be linked to better health (Umberson 1987). However, recent research has shown a changing pattern of relationships, in which 
the health differential between married and never-married men has substantially reduced (Liu and Umberson 2008). Notwithstanding this, the role of family as a health-promoting resource warrants attention (see also Bolin et al. 2003).

Grzywacz and Fuqua (2000) argue that family can provide instrumental support for workers' health. This was evidenced by workers in our sample reporting that their domestic partners prepared healthy food for them, improving their intake of fruit and vegetables and reducing the intake of unhealthy foods. However, family can also indirectly influence health behaviour through applying social control and/or changing people's priorities (Grzywacz and Fuqua 2000). Some of our participants described how pressures from family members or the priority/value placed on family had prompted them to make changes to their lifestyle to improve their health, suggesting that health behaviour can, at least, partially be explained by social processes within the family. Bandura (2004) developed a social cognitive theory to explain the role played by social interactions in the creation of behavioural contracts in relation to health, as well as goal setting, self-efficacy and reinforcement in making and sustaining health behaviour change. The important role played by family (shown in our causal loop diagram) identified workers' family relationships and social networks outside work as being a key leverage point for health. However, previous research also shows that construction workers struggle to maintain positive family relationships and work-family conflict is high, particularly among project-based workers (Lingard and Francis 2004). In this context, broader issues of quality in work-family interaction should be considered in the design and delivery of strategies to promote healthy behaviours.

Our findings identified aspects of the work environment that workers perceived to have an impact on their health. One particularly salient environmental characteristic for workers was the influence of job insecurity and their precarious employment. The links between job insecurity, precarious employment and health are well established in empirical research (Ferrie et al. 2002, Lewchuk et al. 2008). This is consistent with our findings that concerns about job security - driven by the projectbased nature of construction work - negatively impacted the rate of adoption of the health promotion strategies and workers' health, but also drove long and antisocial hours of work. Working regular overtime is a significant risk factor for injuries and illness (Dembe et al. 2005), yet some workers in our sample indicated that they chose to work excessively long hours in order to ensure their financial security when the projects came to an end. They chose to do this despite recognizing that these long hours were detrimental to their health and ability to participate effectively in family life. The project-based nature of construction work is identified in our causal loop diagram as a contributing factor to job insecurity and (indirectly) to long hours and a low rate of health behaviour change at our data collection sites. These relationships suggest that addressing workers' concerns about job security - driven by the project-based nature of work - should be considered as a leverage point for health promotion. Continuity of employment in construction will ultimately depend on the availability of projects and work, particularly in localized areas. However, industry-based redundancy schemes can provide financial security to workers during periods of unemployment. Some organizations managing redundancy funds in Australia also provide extensive well-being and job support services.

The results indicate that long hours perpetuated unhealthy behaviours, particularly in relation to fatigue and physical exhaustion, engaging in healthy lifestyle behaviours and managing the impact of physically demanding work. The effort-recovery (E-R) model developed by Meijman and Mulder (1998) posits that work results in temporary physiological and psychological 'costs' but, after a period of recovery, workers' psychobiological systems stabilize and return to pre-work levels. If recovery opportunities are sufficient, a high workload will not have an enduring adverse impact on 
health. However, insufficient recovery time is significantly related to poor health (Sluiter et al. 2003). If recovery is not sufficient (as evidenced by the experience of physical tiredness and fatigue), workers need to exert additional effort to complete work tasks the following day, resulting in even higher 'costs' and an intensified need for recovery. This downward spiral is likely to be exacerbated when work hours are long as the availability of time for recovery is limited. This effect was evident in our causal loop diagram in which long hours of work increased fatigue and physical tiredness. In turn, this increased the (negative) impact of physical work experienced by workers. As the physical impact of work increased, given the limited recovery time afforded by long hours of work, a vicious spiral effect created ever-increasing levels of fatigue. Breaking this vicious spiral through the provision of sufficient recovery opportunity is therefore a key leverage point for health promotion identified by our analysis.

\section{Towards an ecological understanding of construction workers' health}

Previous research points to the fact that construction workers' health and occupational disability arise from the interplay between workplace risk factors and health behaviours (Van den Berg et al. 2010, Stattin and Järvholm 2005). For example, Arndt et al. (2005) identify musculoskeletal disorders, cardiovascular disease and mental disorders as causes of occupational disability among construction workers in Germany and link these to both occupational risk and lifestyle factors. Oude Hengel et al. (2012) report a combination of occupational and individual factors to predict construction workers' ability and willingness to work until they reach the pension age in the Netherlands (i.e. 65 years).

There is growing recognition that individual and organizational health risk factors interact in complex ways, and that understanding these interactions is important in the framing and design of health promotion interventions (Schulte et al. 2012). However, a comprehensive, integrated theoretical model of how experiences and conditions in the work and non-work environments interact with individual characteristics to shape health has been lacking.

Our results provide an initial attempt to structure the issue of construction workers' health, drawing on the perspectives of construction workers themselves to identify individual and environmental determinants of health behaviour and outcomes. The ecological approach that we took in this research incorporates concepts from multiple theories and disciplines (Glanz and Bishop 2010).

Taking an ecological perspective on workers' health provides the opportunity to draw on multiple behavioural, social and psychological theories to achieve a more holistic understanding of the individual and environmental determinants of workers' health-related behaviour and health outcomes.

The qualitative system dynamics model developed in this research is the first step in trying to 'unpack' and understand how construction workers' health is shaped by individuals' personal characteristics, beliefs and priorities and how these interact with and are shaped by their experiences and conditions in the various settings in which they live and work.

The resulting model provides some clues as to where some of the critical leverage points with the potential to drive behaviour change and health improvement may lie. 


\section{Multi-level, integrated health promotion programmes}

Noblet and LaMontagne (2006) call for more comprehensive approaches to the design of workplace health promotion programmes, which address adverse conditions of work at the same time as workers' health and lifestyle behaviours. This is consistent with policies designed to integrate occupational health and safety programmes with workplace health promotion programmes, such as the US National Institute of Occupational Safety and Health (NIOSH) Total Worker Health program (Sorensen et al. 2011, Schill and Chosewood 2013).

Stokols et al. (1996a, 1996b) observe that health promotion approaches that focus solely on changing lifestyle behaviours often neglect the contextual factors that produce high rates of relapse and attrition once specific periods of intervention come to an end. In contrast, ecological approaches to workplace health promotion are designed to address the complex interdependencies between individual determinants of health and aspects of the physical, social, cultural and organizational work environments (Stokols et al. 2003). An ecological perspective suggests that targeting workers' behaviour without also addressing environmental determinants of health is likely to produce weak or short-lived results (Sallis et al. 2008).

Despite a growing recognition of the importance of adopting an ecological approach to the design of health promotion programmes, a review of the literature suggests that most interventions still focus on individual or interpersonal characteristics rather than institutional, community or policy-focused strategies (Golden and Earp 2012). The results of our analysis suggest the need for more comprehensive approaches to the promotion of construction workers' health. Indeed, the system dynamics model explains our disappointing quantitative results (Lingard and Turner 2015) and goes some way to explaining why targeting individual health behaviours without also addressing multilevel factors of family, workplace and industry will produce limited health improvements.

The research findings suggest that if government policy objectives are to be achieved in relation to population health and well-being, the adoption of an ecological framework to inform the design of holistic health promotion programmes for targeted populations (e.g. construction workers) will be important. The number of levels at which a health promotion programme operates is referred to as its ecological depth (McLaren and Hawe 2005). Thus, programmes with greater ecological depth will yield an effect at multiple levels, e.g. the individual, the workplace and the family environments. The use of a qualitative system dynamics approach can help expose the characteristics of environments that interact with individual characteristics to shape health behaviours and outcomes. In doing so, they have the potential to inform the development of health promotion programmes with greater ecological depth and increased chance of producing lasting benefits.

The research findings also raise important questions about responsibility for construction workers' health. The responsibility for the management of workplace exposures to physical and psycho-social risk factors is clearly articulated in occupational health and safety legislation as the responsibility of an employer. However, if broader concepts of health and well-being are adopted, then individuals, families and other industry stakeholders also have an important role to play in developing integrated behavioural and environmentally-based health promotion programmes (Stokols 1996a, Best et al. 2003). As Schulte et al. (2015, p. e35) writes:

If a higher level of health is pursued, which subsumes health, is aspirational, and includes reaching human potential, then workers surely must actively engage in the process. How the roles of employer versus employee will be distinguished, and what to do about areas of overlap, are critical questions that need to be addressed. In addition, although well-being at work may be primarily an employer's 
responsibility, wellbeing of the worker or workforce is also the responsibility (or at least in the purview) of others in society (e.g. governments, insurance companies, unions, faith-based and nonprofit organizations) or may be affected by nonwork domains. Clearly, the well-being of the workforce extends beyond the workplace, and public policy should consider social, economic, and political contexts.

Thus, a multi-stakeholder, multi-level approach to the design and implementation of synergistic health promotion programmes for the construction industry is warranted (Weiner et al. 2012). In particular, governments and client agencies could usefully consider the role that procurement and the industry's competitive practices play in driving 'brutal rhythms' and the fast pace of construction work that can have negative health impacts for workers (Ajslev et al. 2013). Construction organizations should similarly consider issues of job design, work scheduling and work-family supports that can impact workers' health directly and/ or their ability to engage in healthy-enabling behaviours. Our study also offers insight for researchers in identifying the design and organization of work as a key factor shaping workers' health (and health-related behaviour) in the construction industry.

\section{Conclusions}

In this paper, we analysed qualitative data collected from construction workers and managers during the implementation of a health promotion intervention in the Australian construction industry. A systematic stepwise process was followed to develop a qualitative system dynamics model to identify the forces, influences and feedback loops that emerged from the data analysis as explanations for the adoption of healthy behaviour and/or the state of workers' health. The model identifies environmental and individual determinants of health behaviour and health, and establishes interdependencies and reciprocal relationships between the factors. The model identifies possible leverage points in the industry, workplace and family environments. The research highlights that lifestyle choices are conditioned by multiple levels of environmental influence. However, at the same time, individuals' motivation and behaviour will also likely be shaped by beliefs relating to susceptibility and ability to make change, as well as their progression along a stage of change continuum. The results suggest that workplace health promotion programmes targeting construction may be more effective if they operate at more than one level of intervention simultaneously. Thus, a more holistic understanding of the dynamic relationships between determinants of health and outcomes can inform the development of health promotion programmes that have greater ecological depth.

\section{Limitations and future research}

The research was limited in terms of the collection of data from two worksites in the construction industry in Queensland. Both sites were operated by a single company. Thus, the factors identified in our causal loop diagram may not be applicable in all contexts. Neither do we claim these factors to be comprehensive. The factors contained in our causal loop diagram were drawn from the qualitative data we collected, but it is possible that other relevant factors (and/or linkages between factors) may not have been mentioned by our research participants. Future research should develop causal loop diagrams in qualitative studies in Australia, as well as other jurisdictions. We argue that qualitative system dynamics modelling has inherent value in defining and structuring problems and identifying patterns of influence. However, quantitative simulation models of workers' health behaviour and outcomes may also be helpful in order to evaluate the likely impact of particular 
health programme levers. Future research could potentially develop and test quantitative system dynamics models of construction workers' health. Also, in this research, we did not take a life course perspective, although it is understood that workers' experiences change over their life course. Future research is being developed to adopt a longitudinal cohort design to evaluate the way that factors at multiple levels shape workers' health behaviours and outcomes as they progress through different stages of their work and family lives.

\section{Funding}

This research was funded by the Department of Justice and Attorney General under the Queensland Government 'Healthier. Happier. Workplaces' initiative (previously 'Workplaces for Wellness') and supported by Lendlease.

\section{References}

Abbe, O.O., et al. 2011. Modeling the relationship between occupational stressors, psychosocial/physical symptoms and injuries in the construction industry. International journal of industrial ergonomics, 41 (2), 106117.

Ajslev, J.Z., et al., 2013. Habituating pain: questioning pain and physical strain as inextricable conditions in the construction industry. Nordic journal of working life studies, 3 (3), 195.

Arndt, V., et al. 2005. Construction work and risk of occupational disability: a ten year follow up of 14,474 male workers. Occupational and environmental medicine, 62 (8), 559-566.

Ballinger, C., Yardley, L., and Payne, S., 2004. Observation and action research. In: D.F. Marks and L. Yardley, eds. Research methods for clinical and health psychology. London: Sage, 102-121.

Bandura, A., 2004. Health promotion by social cognitive means. Health education \& behavior, 31 (2), $143-164$.

Best, A., et al., 2003. An integrative framework for community partnering to translate theory into effective health promotion strategy. American Journal of health promotion, 18 (2), 168-176.

Bolin, K., et al., 2003. Investments in social capital - implications of social interactions for the production of health. Social science \& medicine, 56 (12), 2379-2390.

Borsting Jacobsen, H., et al., 2013. Construction workers struggle with a high prevalence of mental distress, and this is associated with their pain and injuries. Journal of occupational and environmental medicine, 55 (10), 1197-1204.

Boschman, J.S., et al., 2014. The impact of common mental disorders on work ability in mentally and physically demanding construction work. International archives of occupational and environmental health, 87 (1), 51-59.

Bowen, P.A., et al., 2014. Occupational stress and job demand, control and support factors among construction project consultants. International journal of project management, 32 (7), 1273-1284.

Brenner, H. and Ahern, W., 2000. Sickness absence and early retirement on health grounds in the construction industry in Ireland. Occupational and environmental medicine, 57 (9), 615-620.

Bronfenbrenner, U., 2009. The ecology of human development: experiments by nature and design. Cambridge, MA: Harvard University Press. Chu, C.,

Driscoll, T., and Dwyer, S., 1997. The health-promoting workplace: an integrative perspective. Australian and New Zealand journal of public health, 21 (4), 377-385. 
Coyle, G., 2000. Qualitative and quantitative modelling in system dynamics: some research questions. System dynamics review, 16 (3), 225-244.

Coyle, G., 2001. Rejoinder to Homer and Oliva. System dynamics review, 17 (4), 357-363.

Dembe, A.E., et al., 2005. The impact of overtime and long work hours on occupational injuries and illnesses: new evidence from the United States. Occupational and environmental medicine, 62 (9), 588-597.

Dong, X.S., et al., 2012. Chronic back pain among older construction workers in the United States: a longitudinal study. International journal of occupational and environmental health, 18 (2), 99-109.

Ettner, S.L. and Grzywacz, J.G. (2001) Workers' perceptions of how jobs affect health: a social ecological perspective. Journal of occupational health psychology, 6 (2), 101-113.

Ferrie, J.E., et al., 2002. Effects of chronic job insecurity and change in job security on self reported health, minor psychiatric morbidity, physiological measures, and health related behaviours in British civil servants: the Whitehall II study. Journal of epidemiology and community health, 56 (6), 450-454.

Galea, S., Riddle, M., and Kaplan, G.A., 2010. Causal thinking and complex system approaches in epidemiology. International journal of epidemiology, 39 (1), 97-106.

Gram, B., et al., 2012. Effect of individualized worksite exercise training on aerobic capacity and muscle strength among construction workers - a randomized controlled intervention study. Scandinavian journal of work, environment and health, 38 (5), 467-75.

Gillen, E.M., et al., 2014. Social ecology of asthma: engaging stakeholders in integrating health behaviour theories and practice-based evidence through systems mapping. Health education \& behavior, 41 (1), 63-77.

Glanz, K. and Bishop, D.B., 2010. The role of behavioral science theory in development and implementation of public health interventions. Annual review of public health, 31, 399-418.

Golden, S.D. and Earp, J.A.L., 2012. Social ecological approaches to individuals and their contexts: twenty years of health education \& behavior health promotion interventions. Health education \& behavior, 39 (3), 364-372.

Groeneveld, I.F., et al., 2010. Sustained body weight reduction by an individual-based lifestyle intervention for workers in the construction industry at risk for cardiovascular disease: results of a randomized controlled trial. Preventive medicine, 51 (3-4), 240-246.

Grzywacz, J.G. and Fuqua, J., 2000. The social ecology of health: leverage points and linkages. Behavioral medicine, $26(3), 101-115$.

Hannertz, H., et al., 2005. Disability retirement among former employeesat the construction of the Great Belt Link. Public health, 119 (4), 301-304. Hirsch, G.B., et al., 2010. A system dynamics model for planning cardiovascular disease preventions. American journal of public health, 100, 616-622.

Homer, J.B. and Hirsch, G.B., 2006. System dynamics modeling for public health: background and opportunities. American journal of public health, 96 (3), 452-458.

Homer, J. and Oliva, R., 2001. Maps and models in system dynamics: a response to Coyle. System dynamics review, 17 (4), 347-355. International Labour Office, 2016. Available from: http://www.

ilo.org/wcmsp5/groups/public/@dgreports/@dcomm/ documents/statement/wcms_095910.pdf [Accessed 5 August 2016].

Joffe, H. and Yardley, L., 2004. Content and thematic analysis. In: D.F. Marks and L. Yardley, eds. Research methods for clinical and health psychology. Sage: London, 56-68.

Kok, G., et al., 2008. The ecological approach in health promotion programs: a decade later. American journal of health promotion, 22 (6), 437-442. 
Kolmet, M., Marino, R., and Plummer, D. 2006. Anglo-Australian male blue-collar workers discuss gender and health issues. International journal of men's health, 5 (1), 81-91.

Kreuter, M.W., et al., 2004. Understanding wicked problems: a key to advancing environmental health promotion. Health education \& behavior, 31, 441-454.

LeBlue, R., et al., 2012. Systems thinking tools as applied to community-based participatory research: a case study. Health education \& behavior, 39 (6), 745-751.

Lewchuk, W., Clarke, M., and de Wolff, A., 2008. Working without commitments: precarious employment and health. Work, employment \& society, 22 (3), 387-406.

Lingard, H. and Francis, V., 2004. The work-life experiences of office and site-based employees in the Australian construction industry. Construction management and economics, 22 (9), 991-1002.

Lingard, H. and Turner, M., 2015. Improving the health of male, blue collar construction workers: a social ecological perspective. Construction management and economics, 33, 18-34.

Lingard, H., Francis, V., and Turner, M., 2010. Work-family conflict in construction: the case for a finer grained analysis. Journal of construction engineering and management, 136 (11), 1196-1206.

Liu, H. and Umberson, D.J., 2008. The times they are a changin': marital status and health differentials from 1972 to 2003. Journal of health and social behavior, 49 (3), 239-253.

Ludewig, P.M. and Borstad, J.D., 2003. Effects of a home exercise programme on shoulder pain and functional status in construction workers. Occupational and environmental medicine, 60 (11), 841-849.

Lynch, J.W., Kaplan, G.A., and Salonen, J.T., 1997. Why do poor people behave poorly? Variation in adult health behaviours and psychosocial characteristics by stages of the socioeconomic lifecourse. Social science \& medicine, 44 (6), 809-819.

Mabry, P.L., et al., 2013. Opening a window on systems science research in health promotion and public health. Health education \& behavior, 40 (suppl. 1), 5S-8S.

Maes, L., et al., 2015. Effectiveness of workplace interventions in Europe promoting healthy eating: a systematic review. European journal of public health, 22 (5), 677-682.

McLaren, L. and Hawe, P., 2005. Ecological perspectives in health research. Journal of epidemiology and community health, $59(1), 6-14$.

Meijman, T.F. and Mulder, G., 1998. Psychological aspects of workload. In: P.J.D. Drenth and H.T. Thierry, eds. Handbook of work and organizational psychology: vol. 2. Work psychology. Hove: Psychology Press, 5-33.

Meltzer, H., et al., 2008. Patterns of suicide by occupation in England and Wales: 2001-2005. British journal of psychiatry, 193 (1), 73-76.

Naaldenberg, J., et al., 2009. Elaborating on systems thinking in health promotion practice. Global health promotion, 16 (1), 39-47.

Noblet, A. and LaMontagne, A.D., 2006. The role of workplace health promotion in addressing job stress. Health promotion international, 21 (4), 346-353.

Oude Hengel, K.M., et al., 2012. Effectiveness of an intervention at construction worksites on work engagement, social support, physical workload and the need for recovery: results from a cluster randomized controlled trial. BMC public health, 12. doi: 10.1186/1471-2458-12-1008.

Peters, D.H., 2014. The application of systems thinking in health: why use systems thinking? Health research policy and systems, 12, 1. 
Petersen, J.S. and Zwerling, C., 1998. Comparison of health outcomes among older construction and blue-collar employees in the United States. American journal of industrial medicine, 34 (3), 280-287.

Pritchard, C. and Dixon, P.B., 2008. Reporting of skin cancer risks in the house-building industry: Alternative approaches to the analysis of categorical data. Public health, 122 (3), 237-242.

Rushton, L., Hutchings, S., and Brown, T., 2008. The burden of cancer at work: estimation as the first step to prevention. Occupational and environmental medicine, 65 (12), 789-800.

Ritchie-Dunham, J.L. and Méndez Galván, J.F., 1999. Evaluating epidemic intervention policies with systems thinking: a case study of dengue fever in Mexico. System dynamics review, 15 (2), 119-138.

Sallis, J.F., Owen, N., and Fisher, E.B., 2008. Ecological models of health behavior. In: K. Glanz, B.K. Rimer, and K. Viswanath, eds. Health behavior and health education: theory, research, and practice, 4th ed., 465-486. San Francisco, CA: Jossey-Bass.

Schill, A.L. and Chosewood, L.C., 2013. The NIOSH total worker health ${ }^{\mathrm{TM}}$ program: an overview. Journal of occupational and environmental medicine, 55, S8-S11.

Schulte, P.A., et al., 2012. Interaction of occupational and personal risk factors in workforce health and safety. American journal of public health, 102 (3), 434-448.

Schulte, P.A., et al., 2015. Considerations for incorporating "wellbeing" in public policy for workers and workplaces. American journal of public health, 105 (8), e31-e44.

Siebert, U., et al., 2001. Demonstration of the healthy worker survivor effect in a cohort of workers in the construction industry. Occupational and environmental medicine, 58 (9), 774-779.

Sluiter, J.K., et al., 2003. Need for recovery from work related fatigue and its role in the development and prediction of subjective health complaints. Occupational and environmental medicine, 60 (suppl. 1), 62 i-70.

Snashall, D., 2005. Occupational health in the construction industry. Scandinavian journal of work, environment and health, 31 (Suppl. 2), 5-10.

Sorensen, G., et al., 2007. Tools for health: the efficacy of a tailored intervention targeted for construction laborers. Cancer causes and control, 18 (1), 51-59.

Sorensen, G., et al., 2011. The role of the work context in multiple wellness outcomes for hospital patient care workers. Journal of occupational and environmental medicine, 53 (8), 899.

Stattin, M. and Järvholm, B., 2005. Occupation, work environment, and disability pension: a prospective study of construction workers. Scandinavian journal of public health, 33 (2), 84-90.

Stocks, S.J., et al., 2011. Occupation and work-related ill-health in UK construction workers. Occupational medicine, 61 (6), 407-415.

Stokols, D., 1992. Establishing and maintaining healthy environments: toward a social ecology of health promotion. American psychologist, 47, 6-22.

Stokols, D., 1996a. Translating social ecological theory into guidelines for community health promotion. American journal of health promotion, 10, 282-298. Stokols, D., Allen, J., and Bellingham, R.L., 1996b. The social ecology of health promotion: implications for research and practice. American journal of health promotion, 10 (4), 247-251.

Stokols, D., et al., 2003. Increasing the health promotive capacity of human environments. American journal of health promotion, 18, 4-13.

Umberson, D., 1987. Family status and health behaviors: social control as a dimension of social integration. Journal of health and social behavior, 306-319. 
Van den Berg, T.I., Elders, L.A., and Burdorf, A., 2010. Influence of health and work on early retirement. Journal of occupational and environmental medicine, 52 (6), 576-83.

Van der Hulst, M., 2003. Long workhours and health. Scandinavian journal of work, environment and health, 29 (3), 171-88.

Vennix, J.A., 1999. Group model-building: tackling messy problems. System dynamics review, 15 (4), $379-401$.

Weeks, M.R., et al., 2013. Multilevel dynamic systems affecting introduction of HIV/STI prevention innovations among chinese women in sex work establishments. Health education \& behavior, 40 (suppl. 1), 111S-122S.

Weiner, B.J., et al., 2012. In search of synergy: strategies for combining interventions at multiple levels. JNCl monographs, 44, 34-41.

Welch, L.S., 2009. Improving work ability in construction workers - let's get to work. Scandinavian journal of work, environment \& health, 35 (5), 321-324.

Wolstenholme, E.F., 1999. Qualitative vs quantitative modelling: the evolving balance. Journal of the operational research society, 50 (S4), 422-428.

Wolstenholme, E.F. and Coyle, R.G. 1983 The development of system dynamics as a methodology for system description and qualitative analysis. Journal of the operational research society, 34 (7), 569-581. 